\title{
Multiplication végétative de Cola acuminata (Pal. de Beauv.) Schott \& Endlicher par marcottage à Kisangani, République Démocratique du Congo
}

\author{
Augustin PALUKU ${ }^{1 *}$, Marcel BWAMA ${ }^{2}$, Albert OKUNGO ${ }^{1}$ et Patrick VAN DAMME ${ }^{3}$ \\ ${ }^{I}$ Institut Facultaire des Sciences Agronomiques de Yangambi, IFA-Yangambi, B.P. 28 Yangambi, RD Congo ; \\ ${ }^{2}$ Université Pédagogique Nationale, UPN, Kinshasa, RD Congo ; \\ ${ }^{3}$ Ghent University, B-9000, Gent, Belgium and Faculty of Tropical Agrisciences, Czech University of Life \\ Sciences, Prague, Czech Republic. \\ "Corresponding author; E-mail: panapasteur2000@gmail.com; Tél.: +243992140413
}

\section{REMERCIEMENTS}

Nous remercions le «World Agroforestry Centre (West and Central Africa, Yaoundé, Cameroun)» qui à travers son projet «AFTP4A » a soutenu financièrement les travaux de recherche.

\section{RESUME}

A Kisangani, le kolatier, Cola acuminata est utilisé à des fins thérapeutiques et sociales et procure des revenus non négligeables aux personnes impliquées dans sa filière. Les produits de kolatier (noix, écorce, feuille) sont obtenus par des récoltes en forêt qui est pourtant menacée. Sa domestication est une nécessité. La présente étude avait pour objet de déterminer le diamètre et l'orientation des branches pour la multiplication végétative de kolatier. La technique de marcottage aérien a été utilisée. Trois classes de diamètre $(2,0-2,9 ; 3,0-$ 3,9 et 4,0-4,9 cm) et deux orientations (plagiotrope et orthotrope) des branches ont été investiguées pour leurs effets sur l'enracinement. Les résultats ont montré que les racines apparaissent à partir de la $6^{\circ}$ semaine après la pose des marcottes. Des taux d'enracinement de $82,2 \pm 5,7 \%$ et $75,5 \pm 6,4 \%$ ont été obtenus avec les branches orthotropes et plagiotropes respectivement. Les différentes classes de diamètre de branches ont donné $76,7 \pm$ $7,8 \% ; 63,3 \pm 8,9 \%$ et $43,3 \pm 9,2 \%$ pour $4,0-4,9 \mathrm{~cm} ; 3,0-3,9 \mathrm{~cm}$ et $2,0-2,9 \mathrm{~cm}$ respectivement. Ces résultats indiquent que $C$. acuminata répond bien à la technique de marcottage, et que les branches orthotropes de diamètre compris entre 4 et $4,9 \mathrm{~cm}$ sont bien indiquées pour un enracinement meilleur des marcottes. La technique de marcottage peut de ce fait être utilisée pour la multiplication conforme des individus élites chez cette espèce.

(C) 2018 International Formulae Group. All rights reserved.

Mots clés : Marcottes, diamètres, orthotrope, plagiotrope, enracinement.

\section{Vegetative propagation of Cola acuminata (Pal. de Beauv.) Schott \& Endlicher by layering in Kisangani, Democratic Republic of Congo}

\begin{abstract}
In Kisangani, kola tree, Cola acuminata is used for healing in folk medicine and ceremonial purposes. It provides significant incomes to people involved in its value chain. Deforestation pattern hinders kola tree products, such as nuts, tree barks, leaves, sustainable harvesting. Hence, kola tree domestication is required. Study aimed to determine kola branch diameter and orientation for vegetative propagation. Air layering was used. Three diameters $(2.0-3 ; 3.0-3.9$ and $4.0-4.9 \mathrm{~cm})$ and two branches orientations (plagiotropic and orthotropic) were investigated for their effects on branches rooting. Results showed that roots appeared six
\end{abstract}


weeks after marcots setting. Rooting percentages of $82.2 \pm 5.7 \%$ and $75.5 \pm 6.4 \%$ were determined for vertical (orthotropic) and horizontal (plagiotropic) branches respectively. The different branch diameters were $76.7 \pm$ $7.8 \%, 63.3 \pm 8.9 \%$ and $43.3 \pm 9.2 \%$ for intervals of $4.0-4.9 \mathrm{~cm} ; 3.0-3.9 \mathrm{~cm}$ and $2.0-2.9 \mathrm{~cm}$ respectively. These results indicate that, for best rooting, C. acuminata responded well to air layering with verticals branches diameters interval between $4.0-4.9 \mathrm{~cm}$. Air layering may be suggested for vegetative multiplication of selected candidate of the species.

(C) 2018 International Formulae Group. All rights reserved.

Keywords: Layers, diameters, orthotropic, plagiotropic, rooting.

\section{INTRODUCTION}

La richesse diversifiée en ressources végétales et animales de la forêt est la raison principale de son exploitation (Carpe, 2001 ; Segers, 2002). La forêt procure aux populations riveraines de la terre fertile pour cultiver, du bois de chauffe, du bois d'œuvre et des produits à valeur alimentaire, commerciale, médicinale et culturelle (Tchatat et Ndoye, 2006).

En République Démocratique du Congo en général et dans la province de la Tshopo en particulier, plusieurs légumes et fruits qui entrent dans les habitudes alimentaires traditionnelles proviennent des plantes locales (Cola acuminata, Annonidium mannii, Gnetum africanum, Talinum triangulare, Myrianthus arboreus, Landolphia owanriensis, Aframomum laurentii, Piper guineensis,....) qui sont d'une importance capitale comme nutriment, potion médicinale, apetissant, tonifiant ou pour les rites coutumiers ( Nyakabwa et al., 1990; Kawukpa et Angoyo, 1994 ; Ekop, 2007 ; Iloh et al., 2009). Des études menées par Everaert (2008), ont montré que les produits d'au moins 15 plantes alimentaires locales sont commercialisés dans les différents marchés de la ville de Kisangani, en province de la Tshopo, et que les noix de $C$. acuminata figurent parmi les 7 les plus demandés. Outre le fait que la forêt tropicale abrite une grande richesse d'arbres fruitiers et de plantes médicinales qui font actuellement l'objet d'un commerce local, régional voire même international, ces espèces sont encore, en grande partie, exploitées dans leurs états naturels (ramassage) et n'ont jamais été étudiées de façon systématique. Très peu d'attention a été accordée à leur domestication ou leur culture, et à l'amélioration de leur base génétique (Degrande et Facheux, 2002). Pourtant, ces plantes subissent des pressions d'exploitation qui les éloignent de plus en plus des agglomérations habitées et se font rares (Bwama et al., 2007). Suite à la déforestation, l'approvisionnement en produits fruitiers locaux pose des problèmes dans des nombreuses contrées du monde (PNUD, 2005).

La population locale aimerait voir améliorer la production de ces espèces qui sont utiles dans leur vécu quotidien (Everaert, 2008). La multiplication végétative, notamment le bouturage, le greffage et le marcottage, reste l'une des techniques les plus appropriées pour la capture et la conservation de traits désirés des arbres fruitiers par les producteurs (Dupriez et De Leener, 1987; Kanmegne et al., 2015).

Etant donné la nécessité urgente de préserver et de développer le potentiel génétique et économique de ces espèces, il serait souhaitable d'envisager un programme de recherche en domestication (Degrande et Facheux, 2002). L'introduction de ces espèces dans les systèmes agricoles pourrait améliorer l'offre en ces produits. Considérant les problèmes de la déforestation accrue, du non mis en culture et le manque de recherches approfondies sur les plantes fruitières locales, nous nous sommes intéressés, à la multiplication végétative par marcottage de Cola acuminata, en vue de la domestiquer dans la région de Kisangani en République Démocratique du Congo. La réponse à la technique de marcottage serait fonction de la dimension et de la position des branches (Mialoundama et al., 2002; Mbete et al., 2011) Les branches vigoureuses, suite à une grande proportion de réserves nutritives, s'enracineraient mieux que les branches minces (Kengue et Tchio, 1994). Les branches de position verticale, orthotrope, par suite d'une bonne exposition à la lumière et donc une activité photosynthétique importante 
influencerait plus l'enracinement que les branches en position horizontale, plagiotrope.

La présente recherche a pour objectif la mise au point d'un protocole de multiplication végétative de $C$. acuminata par la technique de marcottage. Spécifiquement, elle vise à déterminer l'influence de l'orientation et du diamètre des branches sur leur capacité d'enracinement.

\section{MATERIEL ET METHODES Milieu d'étude}

L'étude a été menée à Kisangani, sur des arbres situés à la Faculté des Sciences $\left(404-406 \mathrm{~m}, \quad 0^{\circ} 30^{\prime} 41^{\prime \prime}-0^{\circ} 30^{\prime} 45^{\prime \prime} \mathrm{N}\right.$ et $\left.25^{\circ} 12^{\prime} 20^{\prime \prime}-25^{\circ} 12^{\prime} 31^{\prime \prime} \mathrm{E}\right)$ de l'Université de Kisangani en République Démocratique du Congo. La ville de Kisangani est située dans la partie Nord-Est de la cuvette centrale congolaise. Elle est caractérisée par un climat chaud et humide du type Africain, selon la classification de Köppen. Les températures et les précipitations moyennes annuelles sont respectivement, de $25{ }^{\circ} \mathrm{C}$ et $1800 \mathrm{~mm}$ (Nyongombe, 1993). Robyns (1948) et Ndjele (1988) classent la végétation de Kisangani dans le secteur forestier central de la région guinéenne. Cette végétation est fortement influencée par les activités anthropiques, on y retrouve essentiellement de formations végétales suivantes : les recrus forestiers, les forêts primitives et les forêts rivulaires et marécageuses (Mate, 2001).

\section{Matériel végétal utilisé}

Le matériel végétal était constitué d'arbres en production de l'espèce, $C$. acuminata. C'est un arbre qui peut atteindre $30 \mathrm{~m}$ de hauteur, pour un diamètre du tronc de 30 à $50 \mathrm{~cm}$. Le tronc a des branches proches de la base (Vivien et Faure, 1996; Ngatchou, 2004 ; Lejoly et al., 2010 ).

\section{Méthodologie}

Les arbres sélectionnés pour être marcottés, étaient ceux produisant des fruits (Mialoundama et al., 2002) ayant des branches à ports orthotropes ou plagiotropes et de différents diamètres. Deux expériences ont été menées, la première portant sur l'influence du diamètre des branches et la deuxième portant sur l'influence de l'orientation des branches, sur l'enracinement des marcottes.
Expérience 1: Influence du diamètre de branches sur l'enracinement de marcottes

L'échantillon était constitué de dix arbres sur lesquels neuf marcottes ont été placées par arbre, à raison de trois marcottes par classe de diamètre. Trois classes de diamètre ont été testées : 2,0 - 2,9 cm; 3,0 $3,9 \mathrm{~cm}$ et 4,0 - 4,9 $\mathrm{cm}$ (Mialoundama et al., 2002 ; Tchoundjeu et al., 2002).

Expérience 2: Influence de l'orientation des branches sur l'enracinement de marcottes

L'échantillon était constitué de quinze arbres avec six marcottes par arbre, à raison de trois pour chacune de deux orientations de branches (orthotrope et plagiotrope). Les dimensions de branches variaient de 4 à $6 \mathrm{~cm}$.

Les dispositifs utilisés simulaient les blocs complets randomisés où chaque arbre est considéré comme unité expérimentale.

A la base de chaque rameau retenu, l'écorce a été enlevée à l'aide d'un couteau tranchant sur une longueur de $15 \mathrm{~cm}$ environ. La partie dénudée a été bien grattée pour éliminer le cambium (partie gluante) afin d'éviter que l'écorce ne se reconstitue. Une feuille de film transparent $(40 \times 30 \mathrm{~cm})$ a alors été posée en réceptacle autour du rameau sur la zone dénudée et puis maintenue en place par une ficelle. Le réceptacle a été ensuite chargé avec du substrat de manière à créer un bourrelet recouvrant entièrement la zone dénudée. La sciure de bois bien décomposée a été utilisée comme substrat d'enracinement. La stérilisation s'est faite par d'eau bouillante pour le débarrasser des micro-organismes susceptibles de contaminer la plaie.

\section{Observations et analyses statistiques}

L'enracinement des marcottes a été noté toutes les semaines, en même temps que la possibilité de sevrage. La décision de sevrer une marcotte est prise dès que le faisceau racinaire envahi visiblement le substrat (Figure 1). Les données ont été analysées en utilisant le logiciel SPSS 14.0: pour l'expérience 1, l'analyse de la variance (ANOVA) a été appliquée et le test de Duncan a servi pour déceler les niveaux de significations entre les traitements. Pour l'expérience 2, le test-t a été utilisé pour comparer les moyennes des traitements. Les analyses ont été effectuées au niveau de signification de $5 \%$. 


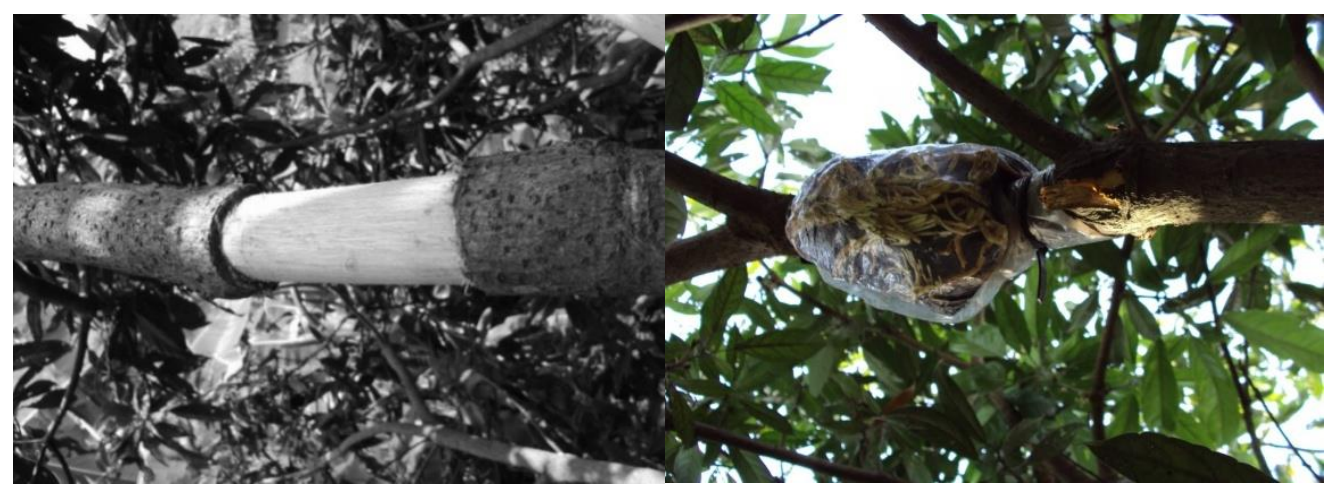

Figure 1: Branche avec écorce dégagée (gauche) et marcotte enracinée (droite) de C. acuminata.

\section{RESULTATS}

Effet du diamètre des branches sur l'enracinement des marcottes

Le taux d'enracinement des marcottes de C. acuminata a été affecté par le diamètre de branches, après 17 semaines d'observation (Figure 2). Le taux d'enracinement a augmenté avec le diamètre de branches. Les branches de diamètre 4,0-4,9 cm ont donné un taux élevé d'enracinement, soit $76,7 \pm 7,8 \%$; contre seulement $63,3 \pm 8,9 \%$ et $43,3 \pm 9,2 \%$ pour les branches de 3,0-3,9 cm et 2,0-2,9 cm respectivement. (Figure 2). Au cours des observations, les taux d'enracinement des marcottes de dimension 4,0-4,9 cm sont restés toujours supérieurs aux autres dimensions. Les différences significatives $(p<0,05)$ sont observées à la $6^{\mathrm{e}}, 16^{\mathrm{e}}$ et $17^{\mathrm{e}}$ semaine ; à la $7^{\mathrm{e}}$ semaine les différences entre les dimensions de branches sont hautement significatives $(p=0,007)$. Le test de Duncan montre des similitudes de taux d'enracinement entre les dimensions 2,0-2,9 $\mathrm{cm}$ et 3,0-3,9 $\mathrm{cm}$; ainsi qu'entre 3,0-3,9 cm et 4,0-4,9 cm (tableau 1).

La plupart des branches enracinées sont observables au $4^{\mathrm{e}}$ mois, $40 \pm 9,1 \%$ pour les branches entre $4,0-4,9 \mathrm{~cm}, 30 \pm 8,5 \%$ pour les branches de dimension entre $3,0-3,9 \mathrm{~cm}$ et
$13 \pm 6,3 \%$ pour les branches entre $2,0-2,9$ $\mathrm{cm}$ (Figure 3).

\section{Effet de l'orientation des branches sur} l'enracinement des marcottes

Le taux d'enracinement a été affecté par la position de branches. Les branches orthotropes ont donné un taux d'enracinement de $82,2 \pm 5,7 \%$, plus élevé que le taux d'enracinement de 75,5 $\pm 6,4 \%$ obtenu avec les branches plagiotropes (Figure 4). L'analyse statistique par le test-t, ne montre pas de différences significatives entre ce deux orientations, en dehors des résultats de la $9^{\mathrm{e}}$ semaine (Tableau 2). La plupart de branches orthotropes se sont enracinées plus précocement que les branches plagiotropes (36\% contre $22 \%$ en 3 mois ; Figure 5). Après 7 mois de marcottage, il avait encore 13,3\% de marcottes plagiotropes qui ne s'étaient pas enracinées contre seulement $4,4 \%$ de branches orthotropes, 13,3 et $15,6 \%$ de marcottes mortes respectivement pour les branches orthotropes et plagiotropes. Si toutes les marcottes orthotropes enracinées ont été sevrées, au moins $6,7 \%$ de marcottes plagiotropes se sont desséchées après s'être enracinées. 


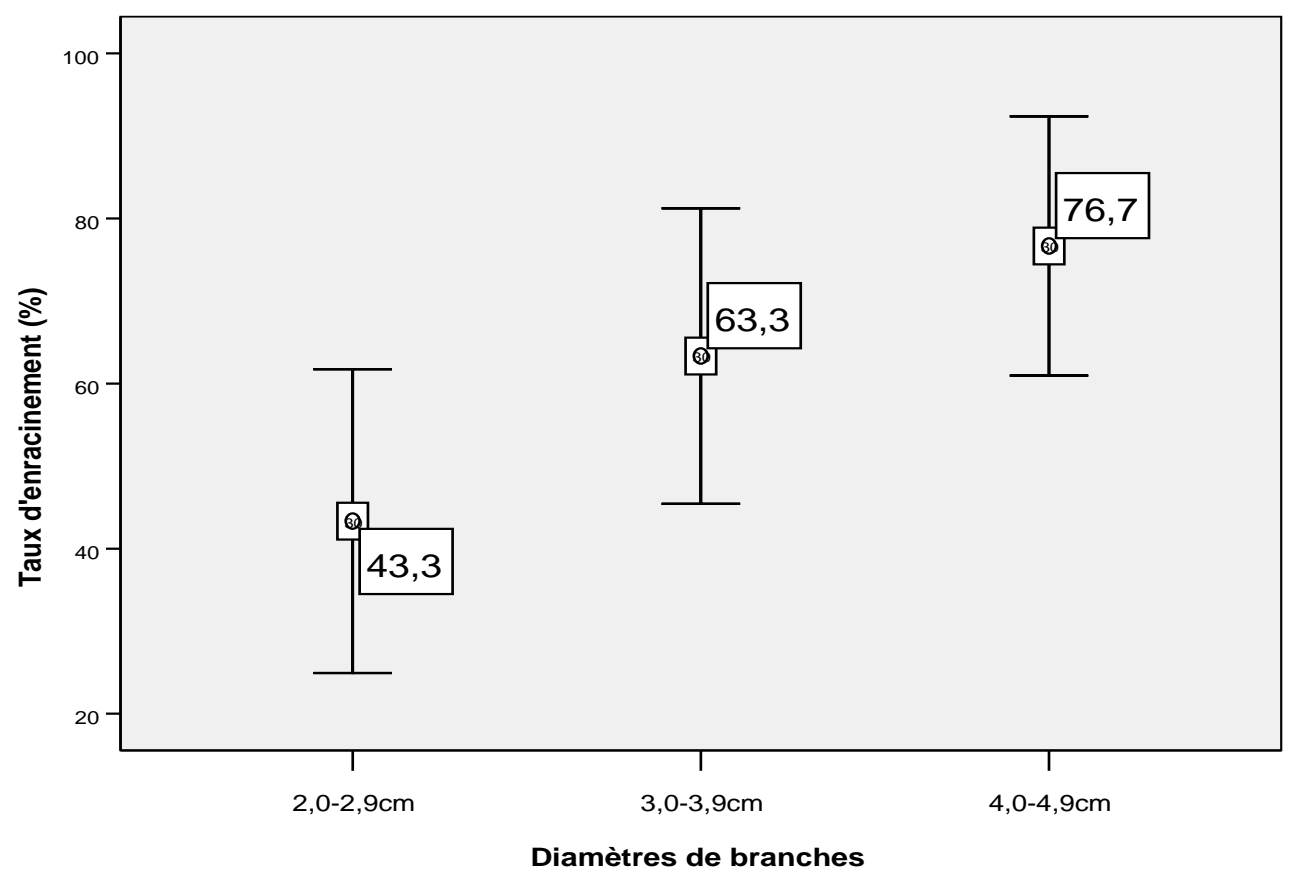

Figure 2: Taux d'enracinement des marcottes de $C$. acuminata en fonction des diamètres de branches.

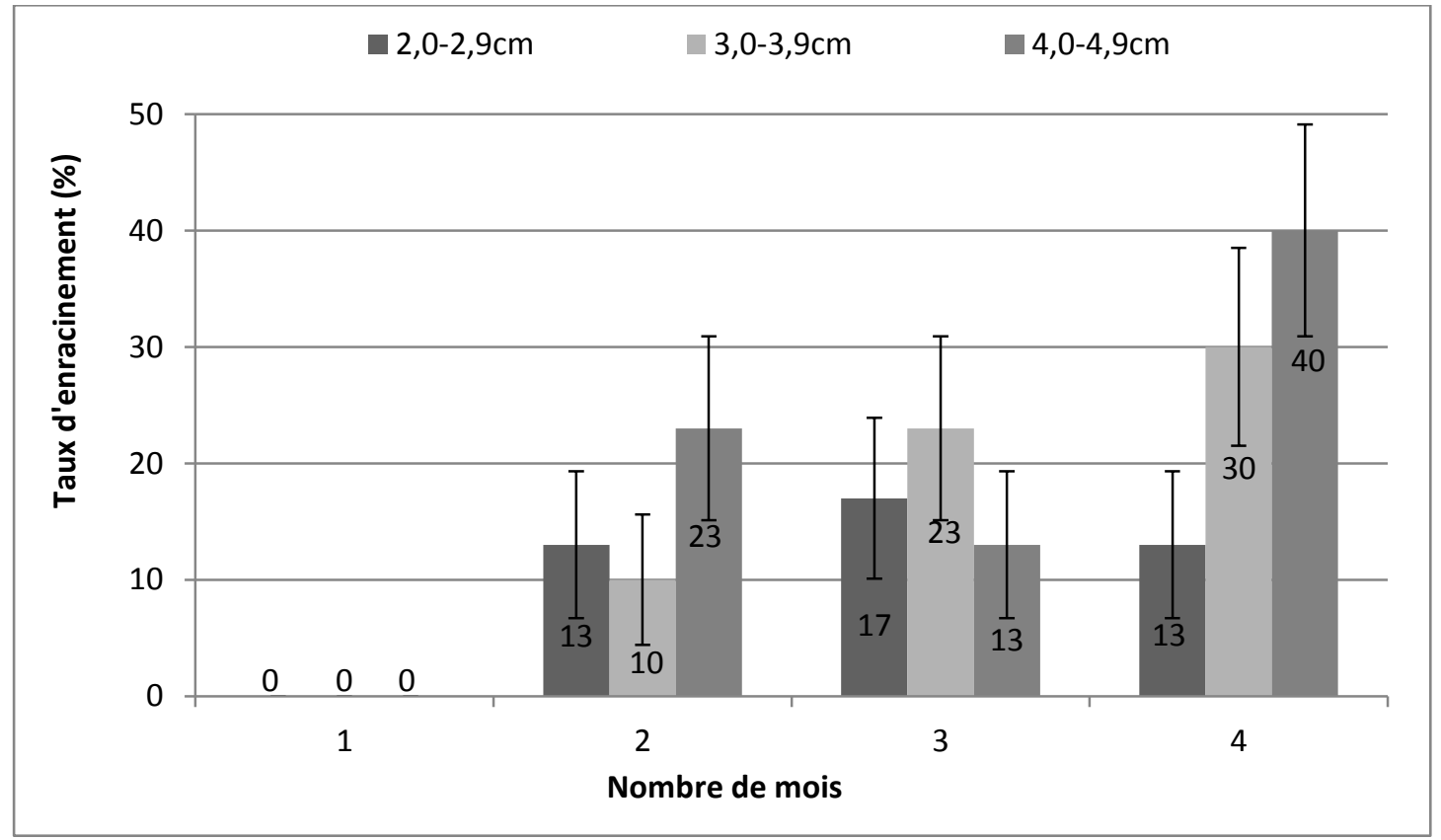

Figure 3: Evolution de l'enracinement des marcottes de $C$. acuminata en fonction des diamètres de branches. 


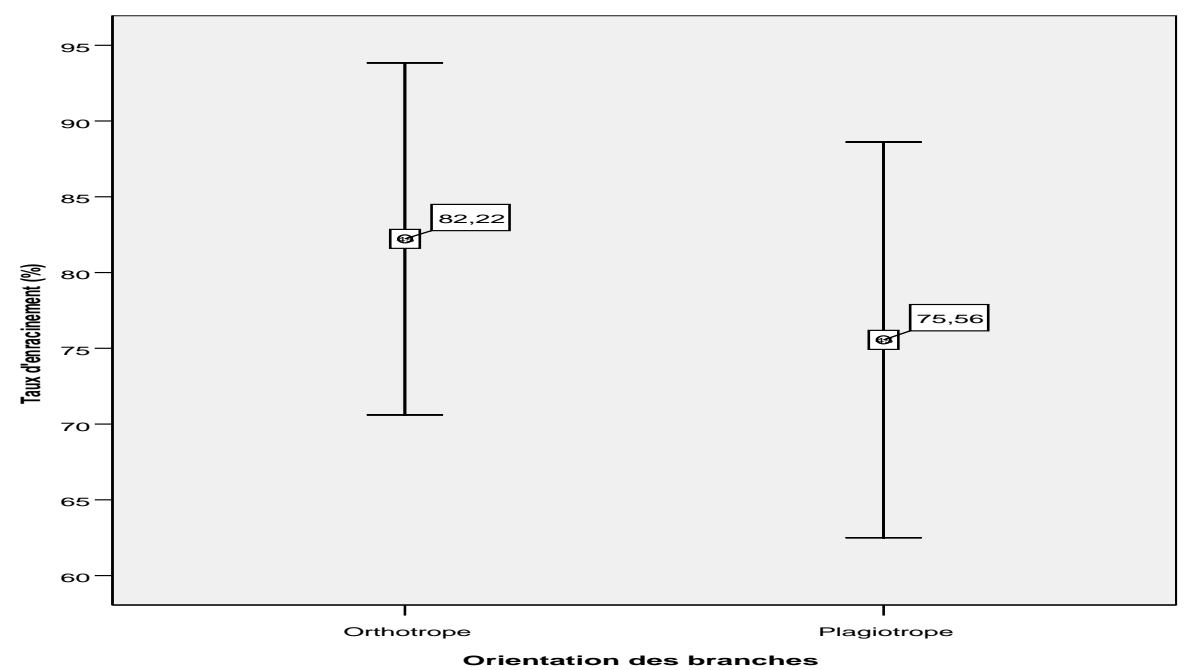

Figure 4: Taux d'enracinement des marcottes de C. acuminata en fonction de l'orientation des branches.

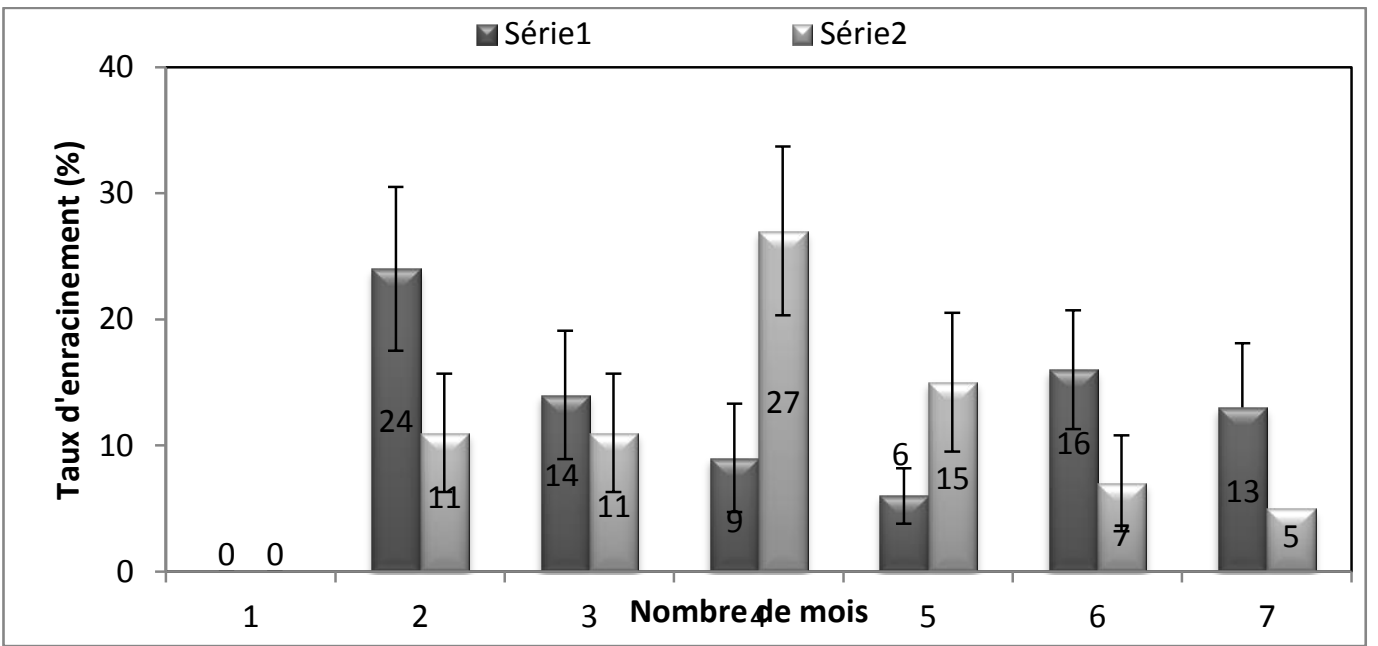

Fugure 5: Fréquence mensuelle d'enracinement des marcottes de $C$. acuminata en fonction de l'orientation des branches.

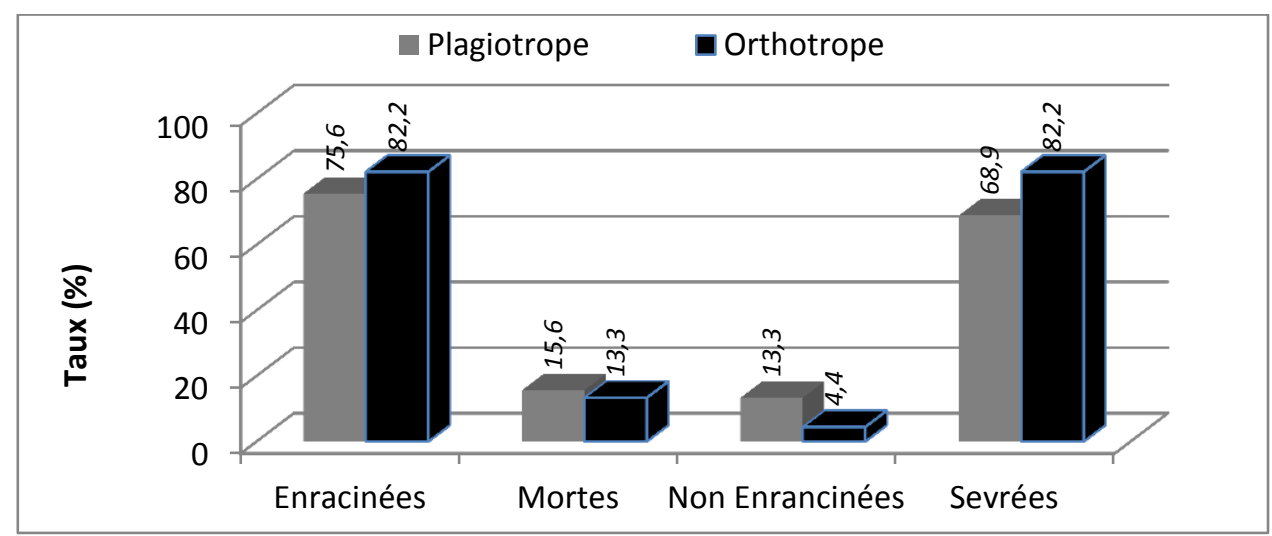

Figure 6: Bilan du marcottage effectué sur C. acuminata en fonction des orientations de branches (situation après 28 semaines). 
A. PALUKU et al. / Int. J. Biol. Chem. Sci. 12(3): 1141-1150, 2018

Tableau 1: Evolution au cours du temps des taux d'enracinement cumulés des marcottes de différentes classes de diamètre de branche de $C$. acuminata.

\begin{tabular}{|c|c|c|c|c|c|c|c|c|c|c|c|c|}
\hline \multirow[b]{2}{*}{ Classes de diamètre } & \multicolumn{12}{|c|}{ Semaines après marcottage } \\
\hline & 6 & 7 & 8 & 9 & 10 & 11 & 12 & 13 & 14 & 15 & 16 & 17 \\
\hline $2,0-2,9 \mathrm{~cm}$ & $00^{\mathrm{a}}$ & $00^{\mathrm{a}}$ & $13 \pm 6,3^{\mathrm{a}}$ & $17 \pm 6,9^{\mathrm{a}}$ & $30 \pm 8,5^{\mathrm{a}}$ & $30 \pm 8,5^{\mathrm{a}}$ & $30 \pm 8,5^{\mathrm{a}}$ & $30 \pm 8,5^{\mathrm{a}}$ & $37 \pm 8,9^{\mathrm{a}}$ & $40 \pm 9,1^{\mathrm{a}}$ & $40 \pm 9,1^{\mathrm{a}}$ & $43 \pm 9,2^{\mathrm{a}}$ \\
\hline $3,0-3,9 \mathrm{~cm}$ & $00^{\mathrm{a}}$ & $03 \pm 3,3^{\mathrm{a}}$ & $10 \pm 5,6^{\mathrm{a}}$ & $20 \pm 7,4^{\mathrm{a}}$ & $27 \pm 8,2^{\mathrm{a}}$ & $27 \pm 8,2^{\mathrm{a}}$ & $33 \pm 8,8^{\mathrm{a}}$ & $37 \pm 8,9^{\mathrm{a}}$ & $43 \pm 9,2^{\mathrm{a}}$ & $50 \pm 9,3^{\mathrm{a}}$ & $63 \pm 8,9^{\mathrm{ab}}$ & $63 \pm 8,9^{\mathrm{ab}}$ \\
\hline $4,0-4,9 \mathrm{~cm}$ & $10 \pm 5,6^{b}$ & $20 \pm 7,4^{b}$ & $23 \pm 7,9^{\mathrm{a}}$ & $27 \pm 8,2^{\mathrm{a}}$ & $33 \pm 8,8^{\mathrm{a}}$ & $33 \pm 8,8^{\mathrm{a}}$ & $37 \pm 8,9^{\mathrm{a}}$ & $53 \pm 9,3^{\mathrm{a}}$ & $63 \pm 8,9^{\mathrm{a}}$ & $67 \pm 8,8^{\mathrm{a}}$ & $77 \pm 7,9^{\mathrm{b}}$ & $77 \pm 7,9^{\mathrm{b}}$ \\
\hline Anova $(p=0,05)$ & 0,045 & 0,007 & 0,341 & 0,635 & 0,858 & 0,858 & 0,865 & 0,168 & 0,100 & 0,115 & 0,13 & 0,028 \\
\hline
\end{tabular}

Les chiffres en gras représentent dans la colonne, les différences significatives par l'Anova. Les valeurs affectées du même lettre ne sont pas significativement différentes après test post hoc de Duncan, $p=0,05$.

Tableau 2: Evolution au cours du temps des taux d'enracinement cumulés des marcottes en fonction de différentes orientations de branches de $C$. acuminata.

\begin{tabular}{|c|c|c|c|c|c|c|c|c|c|c|c|c|c|c|c|c|c|c|c|c|c|}
\hline \multirow[b]{2}{*}{$\begin{array}{l}\text { Orientation } \\
\text { des branches }\end{array}$} & \multicolumn{21}{|c|}{ Semaines après marcottage } \\
\hline & 8 & 9 & 10 & 11 & 12 & 13 & 14 & 15 & 16 & 17 & 18 & 19 & 20 & 21 & 22 & 23 & 24 & 25 & 26 & 27 & 28 \\
\hline Orthotrope & $\begin{array}{l}\text { N } \\
\text { 莫 } \\
\text { in }\end{array}$ & $\stackrel{\omega}{\stackrel{\omega}{+}}$ & 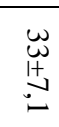 & & $\stackrel{w}{\infty}$ & $\begin{array}{l}\underset{\infty}{\infty} \\
\stackrel{+}{+} \\
\stackrel{\omega}{\omega}\end{array}$ & $\begin{array}{l}\stackrel{+}{N} \\
\stackrel{+}{+} \\
\stackrel{+}{+}\end{array}$ & $\underset{\stackrel{+}{+}}{\stackrel{+}{+t}}$ & 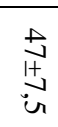 & $\stackrel{u}{\stackrel{u}{+t}}$ & 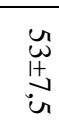 & 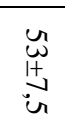 & $\underset{\stackrel{u}{\sim}}{\stackrel{u}{\mid \sim}}$ & 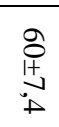 & 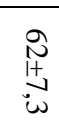 & $\begin{array}{l}\text { Sे } \\
\stackrel{+1}{+} \\
0\end{array}$ & $\begin{array}{l}\stackrel{0}{8} \\
\stackrel{+1}{+} \\
0\end{array}$ & $\begin{array}{l}\text { a } \\
\text { 苦 } \\
\text { in }\end{array}$ & $\begin{array}{l}\infty \\
N \\
\mathbb{N} \\
\infty \\
\infty\end{array}$ & $\begin{array}{l}\infty \\
N \\
\mathbb{N} \\
\infty \\
\infty\end{array}$ & $\begin{array}{l}\infty \\
N \\
\mathbb{N} \\
\stackrel{\infty}{\infty}\end{array}$ \\
\hline Plagiotrope & 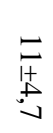 & 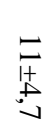 & $\begin{array}{l}\text { No } \\
\text { I+ } \\
\text { O }\end{array}$ & $\begin{array}{l}\text { it } \\
\text { à } \\
\text { w }\end{array}$ & $\begin{array}{l}\text { N } \\
\text { It } \\
\text { 心 }\end{array}$ & $\begin{array}{l}\text { N } \\
\text { 苦 } \\
\text { in }\end{array}$ & 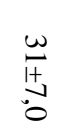 & $\underset{\text { in }}{\stackrel{f}{+t}}$ & 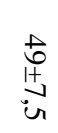 & $\underset{i}{\stackrel{a}{+1}}$ & 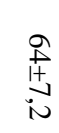 & $\underset{i}{\stackrel{+}{+1}}$ & $\underset{i}{\stackrel{+}{1+}}$ & $\underset{i}{\stackrel{+}{+1}}$ & $\stackrel{9}{+1}$ & 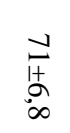 & $\begin{array}{l}\vec{\forall} \\
\stackrel{+}{\alpha} \\
\infty\end{array}$ & 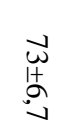 & 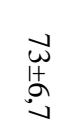 & 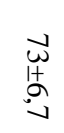 & $\begin{array}{l}\text { वे } \\
\text { it } \\
\text { in }\end{array}$ \\
\hline
\end{tabular}




\section{DISCUSSION}

Les marcottes de branches de Cola acuminata s'enracinent, cet enracinement est fonction du diamètre de branches et de leur orientation. Plus une branche est épaisse, plus elle a la chance de s'enraciner. Cette tendance a déjà été observée sur d'autres plantes principalement sur le safoutier, Dacryodes edulis (Mialoundama et al., 2002; Tchoundjeu et al., 2002). Ces derniers auteurs recommandent respectivement pour cette espèce, les branches de diamètre supérieur à 4 $\mathrm{cm}$ et entre 3 à $5 \mathrm{~cm}$. La vigueur de la branche influence positivement l'enracinement de marcottes (Mialoundama et al., 2002 ; Mbete et al., 2011) par suite de réserves importantes de nutriments sur les grosses branches (Kengue et Tchio, 1994).

Les branches de la position orthotrope (verticale) s'enracinent mieux que celles de la position plagiotrope (horizontale). Ces résultats sont contraires aux observations de Mialoundama et al. (2002) sur D. edulis, où les branches horizontales ont donné plus de marcottes enracinées que les branches verticales. L'orientation de la branche influencerait différemment l'enracinement de marcottes d'une espèce à l'autre. Mbete et al. (2011) faisant les observations sur le marcottage de l'espèce $C$. nitida ont également trouvé les mêmes tendances: les marcottes réalisées sur les rameaux jeunes et ayant un petit diamètre $(1 \mathrm{~cm})$ ont peu de chance de survivre. Le taux d'enracinement est plus élevé sur les rameaux orthotropes que sur les rameaux plagiotropes (24 rameaux orthotropes sur 22 plagiotropes réussies). Ils ont évoqué comme raisons à cette tendance que les rameaux encore jeunes supportent mal la décortication annulaire qui entraine des troubles physiologiques graves et que les rameaux orthotropes ont une morphologie identique au tronc.

Jusqu'à une durée de 7 mois, il y avait encore des marcottes vivantes non enracinées (4 à 13\%) qui pourront s'enraciner par la suite et améliorer les taux d'enracinement. Cette situation peut justifier dans une certaine mesure les différences de taux d'enracinement entre les deux essais : 43 à $77 \%$ dans 4 mois et
76 à $82 \%$ en 7 mois pour les essais 1 et 2 respectivement.

Quel que soit la position ou la dimension de la branche, les marcottes ont commencé à s'enraciner environ 2 mois après la pose. L'enracinement a été observé 6 semaines $(1,5$ mois $)$ après la pose des marcottes, en particulier sur les plus grandes branches $(4,0-4,9 \mathrm{~cm}$ de diamètre). Pour l'espèce $C$. acuminata, les marcottes pratiquées sur les branches orthotropes d'au moins $4 \mathrm{~cm}$ de diamètre ont la capacité d'enracinement la plus élevée.

\section{Conclusion}

L'espèce Cola acuminata se prête au marcottage, et plus de $80 \%$ de marcottes ont donné des racines dans les meilleures conditions de l'expérimentation. L'enracinement commence à partir de la $6^{\mathrm{e}}$ semaine après la pose des marcottes. Les branches les mieux indiquées sont celles de grande taille $(4,0-4,9 \mathrm{~cm}$ de diamètre $)$ et d'orientation verticale. Il serait intéressant de compléter cette étude par l'analyse des effets des autres facteurs tels que la saison, le type de substrat et les traitements auxiniques sur la capacité d'enracinement des marcottes.

\section{CONFLIT D'INTERETS}

Aucun conflit d'intérêts n'est signalé par les auteurs.

\section{CONTRIBUTIONS DES AUTEURS}

Les travaux de recherche sur le terrain ainsi que la rédaction du manuscrit ont été réalisés par $\mathrm{AP}$. $\mathrm{MB}, \mathrm{AO}$ et $\mathrm{PVD}$, promoteurs, ont participé activement à l'élaboration des protocoles de recherche et à la révision du manuscrit.

\section{REMERCIEMENTS}

Nous remercions les ingénieurs Pirwoth Olivier et Akelema Fabrice pour leurs accompagnements dans les réalisations et les suivis techniques des essais sur terrain.

\section{REFERENCES}

Bwama M, Termote C, Dhed'a B, Van Damme P. 2007. Etude préliminaire sur 
la contribution socio-économique de Gnetum africanum dans les ménages de la région de Kisangani. Annales de l'IFA-Yangambi, 1: 117-132.

Carpe. 2001. Congo Basin. Information series.

Taking Action to Manage and Conserve Forest Resources in the Congo Basin. Results and Lessons Learned from the First Phase (1996-2000).

Degrande A, Facheux C. 2002. Production et Commercialisation des plantes produites dans les pépinières villageoises. Guide pratique pour formateurs, encadreurs et pépiniéristes. ICRAF- West and Central Africa. World Agroforestry Centre, p. 52.

Dupriez H, De Leener P. 1987. Jardins et vergers d'Afrique. Ed. Terres et Vie, Nivelles, Belgique, p. 354.

Ekop AS. 2007. Determination of Chemical Composition of Gnetum africanum (AFANG) Seeds. Pakistan Journal of Nutrition, 6(1): 40-43. DOI: 10.3923/pjn.2007.40.43.

Everaert G. 2008. Recherche Ethnobotanique : Etude de marché sur les Plantes Alimentaires Sauvages (R.D. Congo, Province Orientale). Résumé de Thèse. Université de Gent, Gent, Belgique, p. 31.

Iloh AC, Isu NR, Kuta DD. 2009. In vitro callus initiation of a 'threatened' Nigerian leafy vegetable, Gnetum africanum (WILW). Int. J. Biodvers. Conserv., 1(4): 081-086. http://www.academicjournals.org/article/ article1379514273_Iloh et al.pdf.

Kanmegne G, Anouma'a M, Mbibong DA, Mbouobda HD, Fotso, Omokolo DN. 2015. Domestication of Cola anomala K. Schum. (Sterculiaceae): Amenability to vegetative propagation. Forests, Trees and Livelihoods, 24(1): 18-26. DOI: http://dx.doi.org/10.1080/14728028.2014 .941415

Kawukpa U, Angoyo M. 1994. Plantes utiles chez les Batiabetuwa de l'Ile de Mbie, Kisangani, Zaïre. African Study Monographs, 15(2): 49-68. URI: http://hdl.handle.net/2433/68120
Kengue J, Tchio F. 1994. Essais de bouturage et de marcottage de Safoutier (Dacryoides edulis). In : Le Safoutier the African Pear, Kegu J, Nya Ngatchou J (eds). Actes du Séminaire régional sur la valorisation du Safoutier , 4-6 octobre 1994, Douala, 80 - 98pp.

Lejoly J, Ndjele MB, Geerink D. 2010. Catalogue - Flore des plantes vasculaires des Districts de Kisangani et de la Tshopo (RDCongo). $4^{\mathrm{e}}$ éd. Revue et augmentée, incluant les clés et la distribution pour 70 familles. $L a$ Taxonomania, $30: 1-308$.

Mate M. 2001. Croissance de phytomasse et mineralomasse des haies des légumineuses améliorantes, en culture en allée à Kisangani (RDC). Thèse de Doctorat, Faculté des Sciences, Université Libre de Bruxelles, Bruxelles, Belgique, p. 204.

Mbete P, Makoso S, Lelou B, Douh C, Ngokaka C. 2011. Essai de multiplication végétative du Colatier (Cola nitida) via la technique du marcottage au Congo Brazzaville. J. Appl. Biosc., 37: 2485-2490. http://www.m.elewa.org/JABS/2011/37/ 9.pdf

Mialoundama P, Avana ML, Youmbi E, Mapouya PC, Tchoundjeu Z, Mbeuyo M, Galamo GR, Bell JM, Kopguep F, Tsobeng AC, Abega J. 2002. Vegetative propagation of Dacryodes edulis (G.Don) H.J. Lam by marcots, cuttings and micropropagation. Forests, Trees and Livelihoods 12: 85-96. https:// doi.org/10.1080/14728028.2002.975241 2

Ndjele MB. 1988. Les éléments : phytogéographiques et endémiques dans la flore vasculaire du Zaïre. Thèse de Doctorat, Université Libre de Bruxelles, Bruxelles, Belgique, p. 528.

Ngatchou SI. 2004. Etat de la culture paysanne de Cola spp. dans les départements de Boyo et de Bui, Province du Nord - ouest. Mémoire, Inédit, Faculté d'Agronomie et des 
Sciences Agricoles, Université de Dschang, Dschang, Cameroun, p. 87.

Nyakabwa M, Bola M, Vasolene K. 1990. Plantes sauvages alimentaires chez les Kumu de Masako à Kisanagni (Zaïre). African Study Monagraphs, 11(2): 7586.

URI :

http://hdl.handle.net/2433/68064

Nyongombe U. 1993. Contribution à l'étude écologique et biologique des poissons de la rivière MASINDULA (affluent de la Tshopo) à Kisangani. Thèse de Doctorat, IFA-Yangambi, Yangami, RD Congo, p. 193.

Programme des Nations Unies pour le Développement 2005. Diagnostic du dispositif institutionnel d'intervention de l'Etat en matière de gestion économique et financière publique dans la perspective de la décentralisation de la Province Orientale. Rapport de mission d'appui au Programme National d'Urgence et de Renforcement des Capacités, PNUD DAES/ZAI/00/004, Kisangani, RD Congo.
Robyns W. 1948. Les territoires phytogéographiques du Congo belge et Rwanda Urundi. In Atlas Général du Congo Belge. Inst. EAF, Col. Bel. : Bruxelles ; 10.

Segers JM. 2002. Forces motrices déterminant la foresterie à l'horizon 2020. ORGUT Consulting AB, BAD, Abidjan.

Tchatat M, Ndoye O. 2006. Étude des produits forestiers non ligneux d'Afrique centrale : réalités et perspectives. Bois et Forêts des Tropiques, 289(3): 27-39. http://bft.cirad.fr/cd/BFT_289_27-39.pdf

Tchoundjeu Z, Kengeu J, Leakey RRB. 2002. Domestication of Dacryodes edulis: State-of-the-Art. Forests, Trees and Livelihoods, 12: 3-13. https:// doi.org/10.1080/14728028.2002.975240 7

Vivien J, Faure JJ. 1996. Fruitiers Sauvages d'Afrique (Espèces du Cameroun). Ed. Nguila-Kerou, les presses de l'imprimerie Téqui Saint-cénéré, p. 416. 